\title{
Role of JNK signalling pathway and platelet-lymphocyte aggregates in myocardial ischemia-reperfusion injury and the cardioprotective effect of ischemic postconditioning in rats
}

\author{
FAXIN REN ${ }^{1 *}$, NAN MU $^{2 *}$, MINGXIAO GAO $^{1 *}$, JING SUN $^{1}$, CHUANHUAN ZHANG $^{1}$, XIAOJIAN SUN ${ }^{1}$, \\ LIUDONG LI ${ }^{1}$, JUN LI ${ }^{1}$, TONG LIU ${ }^{3}$, GARY TSE ${ }^{4,5}$ and MEI DONG ${ }^{1}$ \\ Departments of ${ }^{1}$ Cardiology and ${ }^{2}$ Gynaecology, The Affiliated Yantai Yuhuangding Hospital of Qingdao University, \\ Yantai, Shandong 264000; ${ }^{3}$ Tianjin Key Laboratory of Ionic-Molecular Function of Cardiovascular Disease, \\ Department of Cardiology, Tianjin Institute of Cardiology, Second Hospital of Tianjin Medical University, \\ Tianjin 300211; ${ }^{4} \mathrm{Li}$ Ka Shing Institute of Health Sciences; ${ }^{5}$ Department of Medicine and Therapeutics, \\ Faculty of Medicine, Chinese University of Hong Kong, Hong Kong, SAR, P.R. China
}

Received March 11,2018; Accepted August 30, 2018

DOI: $10.3892 / \mathrm{mmr} .2018 .9545$

\begin{abstract}
In myocardial ischemia-reperfusion injury (MIRI), increased activity of the c-Jun N-terminal kinase (JNK) pathway and the activation of platelets that leads to the formation of platelet-leukocyte aggregates (PLAs) have been observed. It was hypothesized that ischemic postconditioning in MIRI exerts cardioprotective effects by altering JNK activity, which in turn leads to reduced PLA levels. A total of 60 rats were randomly divided into 6 groups $(n=10$ for each group): i) Control; ii) ischemia-reperfusion injury alone; iii) ischemia-reperfusion with postconditioning (PostC group), iv) treatment with the JNK inhibitor-SP600125; v) postC and treatment with anisomycin; and vi) treatment with the JNK activator-anisomycin. Subsequently, the levels of PLA, infarct size, myocardial injury markers (creatinine kinase-muscle/brain and troponin I) and were measured. Western blotting was used to determine the protein expression of phosphorylated-JNK. MIRI led to increased myocardial infarct size that was associated with raised troponin I and creatine kinase-muscle/brain.
\end{abstract}

Correspondence to: Dr Mei Dong, Department of Cardiology, The Affiliated Yantai Yuhuangding Hospital of Qingdao University, 20 Yuhuangding East Road, Zhifu, Yantai, Shandong 264000, P.R. China

E-mail: dongmei0212@126.com

Dr Gary Tse, Department of Medicine and Therapeutics, Faculty of Medicine, Chinese University of Hong Kong, 30-32 Ngan Shing Street, Hong Kong, SAR, P.R. China

E-mail: tseg@cuhk.edu.hk

${ }^{*}$ Contributed equally

Key words: myocardial ischemia reperfusion injury, ischemia postconditioning, platelet-leukocyte aggregation, c-Jun N-terminal kinase, mitogen-activated protein kinase
At different time points of MIRI, the level of PLA gradually increased. Compared with the injury-reperfusion group, the level of PLA in the PostC and Inhibitor-JNK groups was significantly reduced at $60 \mathrm{~min}$ and $3 \mathrm{~h}$ following reperfusion. MIRI was able to increase the expression of phosphorylated JNK. These effects were significantly reduced by ischemic postC or by treatment with SP600125. By contrast, the addition of anisomycin attenuated these protective effects. JNK is a critical mediator of MIRI. Ischemic postC can reduce the level of PLA during reperfusion by inhibiting the phosphorylation of JNK MAPK, thereby reducing MIRI. Pharmacological inhibition and activation of JNK can improve and reduce cardioprotective effects, respectively. These results explained the mechanism of the cardioprotection of postC and provided novel insight and target for the therapeutic strategy of MIRI.

\section{Introduction}

Myocardial ischemia-reperfusion injury (MIRI) frequently occurs after the ischemic myocardium is treated. When MIRI occurs, the degree of myocardial damage is aggravated and the infarct area expands. The inflammatory response is one of the main factors causing reperfusion injury following acute myocardial ischemia (1). Inflammation is modulated by a number of factors including inflammatory mediators and inflammatory cells that promote myocardial damage from ischemia injury to reperfusion injury.

In acute myocardial infarction (AMI), platelets are activated and can interact with leukocytes, thereby forming platelet-leukocyte aggregates (PLAs). Increased PLA levels may be an indicator of thrombus burden and pro-inflammatory response (2) and has been observed in a variety of cardiovascular diseases, including unstable angina and AMI $(3,4)$. Reperfusion therapy is the cornerstone for salvaging the myocardium, but it can induce damage to the working myocardium via ischemia-reperfusion (I/R) injury. Postconditioning, defined as brief periods of reperfusion alternating with re-occlusion applied during the very early min of reperfusion, 
has been demonstrated to be protective against MIRI $(5,6)$ and therefore may reduce myocardial infarct size, but its underlying mechanism is incompletely elucidated.

The c-Jun N-terminal kinase (JNK) is a member of the mitogen activated protein kinase (MAPK) superfamily. Previous experiments have demonstrated the role of the JNK signaling pathway by increasing JNK expression in MIRI (7-9). However, whether postischemic conditioning exerts its cardio-protective effects by altering the JNK signalling pathway and the levels of PLA have not been examined.

In the present study, a rat model of myocardial ischemia reperfusion in vivo was established. The expression of phosphorylated P38 MAPK and PLA was observed following postconditioning treatment, and the effect was investigated using specific inhibitors of P38 MAPK (SP 600125) and as well as activators of P38 MAPK (anisomycin). Furthermore, the present study aimed to investigate the role of P38 MAPK in signal transduction of postconditioning, as well as its effect on PLA expression.

\section{Materials and methods}

Materials. A total of 60 male Sprague Dawley rats (8 weeks old; weight, 250-280 g) were purchased from Beijing Wei Tong Li Hua Experimental Animal Co., Ltd. (Beijing, China). The rats were housed in an environment with a maintained temperature of $22^{\circ} \mathrm{C}$, a relative humidity of $50 \pm 15 \%$ and a 12-h light/dark cycle. All rats had free access to standard chow and water. The present study conformed to the Guide for the Care and Use of Laboratory Animals published by the US National Institutes of Health (NIH publication no. 85-23; revised 1996) and was approved by the Research Commission on Ethics of the Affiliated Yantai Yuhuangding Hospital of Qingdao University (Yantai, China). For measuring markers of myocardial damage, creatine kinase-muscle/brain (CK-MB) and troponin (TnI) kits were purchased from Merck KGaA (Darmstadt, Germany). The erythrocyte lysate was purchased from BD Biosciences, Franklin Lakes, (NJ, USA). Cluster of differentiation (CD)45 and CD41a were purchased from eBioscience; Thermo Fisher Scientific, Inc. (Waltham, MA, USA). The rabbit anti-mouse phosphorylated JNK (phospho-JNK MAPK/p-JNK) polyclonal antibody was purchased from Santa Cruz Biotechnology, Inc., Dallas, TX, USA and the corresponding secondary antibody was from Jackson ImmunoResearch Laboratories, Inc. (West Grove, PA, USA).

Myocardial ischemia reperfusion model. The rats were anesthetized with an initial intra-peritoneal injection of sodium pentobarbital $(0.23 \mathrm{ml} / 100 \mathrm{~g})$ and were then intubated and ventilated using a rodent respirator. A ligature was inserted under the anterior descending branch of the left coronary artery (LAD), and the model was constructed by ligating the LAD for $30 \mathrm{~min}$ and then loosening the knot of the LAD. Different drugs, including DMSO (0.8 ml; 10\%), SP600125 (6 mg/ $\mathrm{kg}$ in $0.8 \mathrm{ml}$ of $10 \%$ DMSO) and anisomycin $(2 \mathrm{mg} / \mathrm{kg}$ in $0.8 \mathrm{ml}$ of $10 \% \mathrm{DMSO}$ ) were injected into the right jugular vein 5 min following reperfusion. Then 3-4 ml blood was drawn from the carotid artery cannula and different treatments were given according to the measured indexes.
Experimental protocols. A total of 60 rats were randomly allocated to one of the 6 groups $(n=10)$ as follows: i) The sham group; ii) ischemia-reperfusion (I/R) group; iii) post-conditioning (PostC) group; iv) JNK inhibitor SP600125 (I-JNK) group; v) anisomycin/Ani plus postconditioning (Ani + PostC) group and vi) anisomycin (Ani) group (Fig. 1).

In the sham group, the left coronary artery was isolated and threaded but not ligated and left for $30 \mathrm{~min}$. A total of $0.8 \mathrm{ml}$ $10 \%$ DMSO was injected into the right jugular vein $25 \mathrm{~min}$ following ischemia. In the I/R group, $0.8 \mathrm{ml} 10 \%$ DMSO was injected into the right jugular vein $25 \mathrm{~min}$ following ischemia. Following the end of ischemia, continuous reperfusion was resumed and performed for $3 \mathrm{~h}$. In the postconditioning (PostC) group, $0.8 \mathrm{ml}$ of $10 \%$ DMSO into the right jugular vein 25 min following ischemia. After the ischemia was completed, 3 cycles of $1 / 1 \mathrm{~min}$ reperfusion/reocclusion began immediately at the onset of reperfusion and then continuous reperfusion was performed for $3 \mathrm{~h}$. In the I-JNK group, SP600125 (Merck $\mathrm{KGaA} ; 6 \mathrm{mg} / \mathrm{kg}$ in $0.8 \mathrm{ml}$ of $10 \%$ DMSO) was injected into the right jugular vein 25 min following ischemia. Following the end of ischemia, continuous reperfusion was resumed and performed for $3 \mathrm{~h}$. In the Ani + PostC group, anisomycin ( $2 \mathrm{mg} / \mathrm{kg}$ in $0.8 \mathrm{ml}$ of $10 \%$ DMSO) was injected into the right jugular vein $25 \mathrm{~min}$ following ischemia. At the end of ischemia, 3 cycles of $1 / 1 \mathrm{~min}$ reperfusion/reocclusion began immediately at the onset of reperfusion and continuous reperfusion was performed for $3 \mathrm{~h}$. In the Ani group, anisomycin (Merck $\mathrm{KGaA} ; 2 \mathrm{mg} / \mathrm{kg}$ in $0.8 \mathrm{ml}$ of $10 \%$ DMSO) was injected into the right jugular vein 25 min following ischemia. Following the end of ischemia, continuous reperfusion was resumed and performed for $3 \mathrm{~h}$.

Release of serum markers. The serum levels of TnI and CK-MB were analyzed using an automatic biochemistry analyzer with the CK-MB kit. All experiments were carried out according to the manufacturer's protocol.

Measurement of infarct size. A total of $3 \mathrm{~h}$ following reperfusion, the hearts from the rats were excised and maintained at $-20^{\circ} \mathrm{C}$ for $20 \mathrm{~min}$. The hearts were sliced into four sections (thickness, $2 \mathrm{~mm}$ ) from the base to the apex for staining with triphenyltetrazolium chloride $(1 \%)$ at $37^{\circ} \mathrm{C}$ for $15 \mathrm{~min}$ for measuring the area of necrosis. The myocardium of the infarct area was pale, while the active myocardium was red in color. The infarct size was expressed as the ratio of the infarct area to the total left ventricular volume.

Measurement of PLA. Flow cytometry was used to detect the level of PLA as described previously (10). Briefly, sodium citrate anticoagulant was added to $200 \mu \mathrm{l}$ of blood followed by centrifugation at $1,000 \mathrm{~g}$ for $5 \mathrm{~min}$ at room temperature. The supernatant was discarded. A total of 6-10 times the cell volume of red blood cell lysate was added and then gently mixed. A total of 1-2 min post-mixing, the cells were lysed using Red Blood Cell Lysis Buffer (cat. no. 3702; Beyotime Institute of Biotechnology, Shanghai, China). This step was repeated until cracking was complete. Then, PBS was added. The precipitate was resuspended and centrifuged at $1,000 \mathrm{~g}$ for $3 \mathrm{~min}$. The supernatant was discarded. A total of $200 \mu \mathrm{l}$ paraformaldehyde (2\%) was added and fixed for $60 \mathrm{~min}$ at room temperature. PBS 


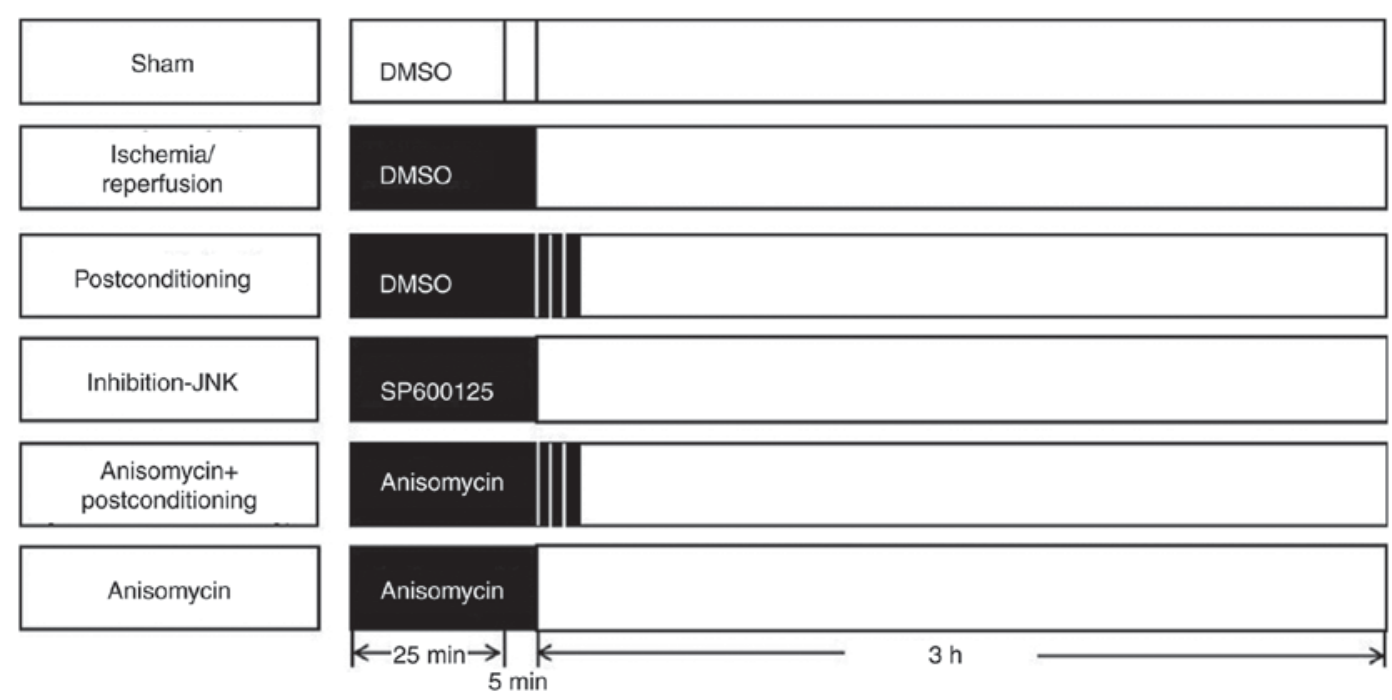

Figure 1. Time-lines of the experimental protocols. The black area represents ischemia for $30 \mathrm{~min}$, the white area represents reperfusion for $3 \mathrm{~h}$. JNK, c-Jun $\mathrm{N}$-terminal kinase.

$(1,600 \mu \mathrm{l})$ was added for $15 \mathrm{~min}$, and centrifuged at $1,000 \mathrm{x} \mathrm{g}$ for $15 \mathrm{~min}$ at room temperature followed by resuspension with PBS for 3 times, and finally dissolved in $200 \mu$ l PBS. To each group of two tubes, CD45 (cat. no. 11-0461-82; $0.25 \mu \mathrm{g} /$ test) and CD41-phycoerythrin (cat. no. PA5-79526; $1 \mu \mathrm{g} / 1 \times 10^{6}$ cells) were used, maintained in a dark room for $30 \mathrm{~min}$ and centrifuged at $1,000 \mathrm{x} \mathrm{g}$ at $4^{\circ} \mathrm{C}$ for $5 \mathrm{~min}$. PBS suspension was detected using a BD FACSCanto II flow cytometer and BD FACSDiva $^{\mathrm{TM}}$ software (BD Biosciences).

Measurement of $p-J N K M A P K$. Western blot analysis was performed as previously described(11). Briefly, the left ventricular myocardium was homogenized in radioimmunoprecipitation assay lysis buffer [hepes (20 mmol/1; $\mathrm{pH} 7.7), \mathrm{MgCl}_{2} 2.5 \mathrm{mmol} / \mathrm{l}$, EDTA $(0.1 \mathrm{mmol} / \mathrm{l})$, B-glycerophosphate $(20 \mathrm{mmol} / \mathrm{l})$, dithiothreitol $(0.5 \mathrm{mmol} / \mathrm{l})$, sodium orthovanadate $(0.1 \mathrm{mmol} / \mathrm{l}), \mathrm{NaCl}$ (75 mmol/l), leupeptin ( $4 \mu \mathrm{g} / \mathrm{ml})$, phenyl-methylsulfonyl fluoride $(20 \mu \mathrm{g} / \mathrm{ml})$ and Triton X-100 (0.05\%; v/v)]. To quantify protein levels, equal amounts of total protein $(10 \mu \mathrm{g})$ were loaded into lanes. Protein concentration was determined using the bicinchoninic acid method (cat. no. A53225; Pierce; Thermo Fisher Scientific, Inc.). After the separation of proteins via SDS-PAGE gel $(10 \%)$ electrophoresis, the proteins were transferred to nitrocellulose membranes and incubated with antibodies against p-JNK MAPK (cat. no. sc-6254; $1: 200$ ) and $\beta$-actin (cat. no. sc-47778; 1:200; both Santa Cruz Biotechnology, Inc., Santa Cruz, CA, USA) for $4 \mathrm{~h}$ at room temperature, which was followed by incubation with horseradish peroxidase-conjugated secondary antibodies (cat. no. 016-030-084; 1:1,000; Jackson ImmunoResearch Laboratories, Inc.) for $1 \mathrm{~h}$ at room temperature. The antigen-antibody complexes were visualized using enhanced chemiluminescence (Beyotime Institute of Biotechnology) at room temperature. The integrated optical density (IOD) of the bands were analysed using Image Pro Plus image analysis software (version 4.1; Media Cybernetics, LP, USA). The IOD was calculated by multiplying the value of the average optical density by area. The ratio of IOD values of the target protein and $\beta$-actin was used to reflect the relative level of the target protein.
Statistical analysis. The data were analysed using the statistical software package SPSS 20.0 (IBM Corp., Armonk, NY, USA) for Windows. The values are expressed as the mean \pm standard error of the mean. A one-way analysis of variance followed by the SNK post hoc test or the Student's t-test was used as appropriate to determine the differences between groups. $\mathrm{P}<0.05$ was considered to indicate a statistically significant difference. All experiments were performed in triplicate.

\section{Results}

Infarct size and serum markers of myocardial damage. Initial experiments examined infarct size following AMI with the infarcted myocardium stained white and the viable myocardium stained red (Fig. 2). Compared with the sham group, the infarct size was $42.2 \%$ in the I/R injury group. The infarct size was significantly reduced in the postconditioning group $(\mathrm{P}<0.05)$ and also following application of the JNK inhibitor, SP600125 (I-JNK). Furthermore, this reduction in infarct size was abolished when the JNK activator, Ani was applied, but not when the hearts underwent postconditioning (Table I).

In the sham group the levels of CK-MB and TnI were $166 \pm 33 \mathrm{pg} / \mathrm{ml}$ and $7.8 \pm 0.5$, respectively (Table I). Compared with the Sham group, the levels of CK-MB and TnI were significantly increased in the $I / R$ injury group $(\mathrm{P}<0.05)$. Compared with the I/R injury group, the levels of CK-MB and TnI were significantly lower in the postconditioning group $(\mathrm{P}<0.05)$, and the JNK inhibitor SP600125, exhibited a similar cardioprotective effect as ischemic postconditioning. By contrast, when the JNK activator, Ani was applied, both CK-MB and TnI were high and not significantly different from those of the I/R group. However, ischemic postconditioning partially protected against myocardial damage even when anisomycin was applied.

Levels of the PLAs and p-JNK. The level of PLAs in the Sham group was $8.70 \pm 0.56,8.99 \pm 0.56,8.80 \pm 0.36$ and $10.11 \pm 0.87$ at $30 \mathrm{~min}$ following ischemia, and at $30,60 \mathrm{~min}$ and $3 \mathrm{~h}$ following 

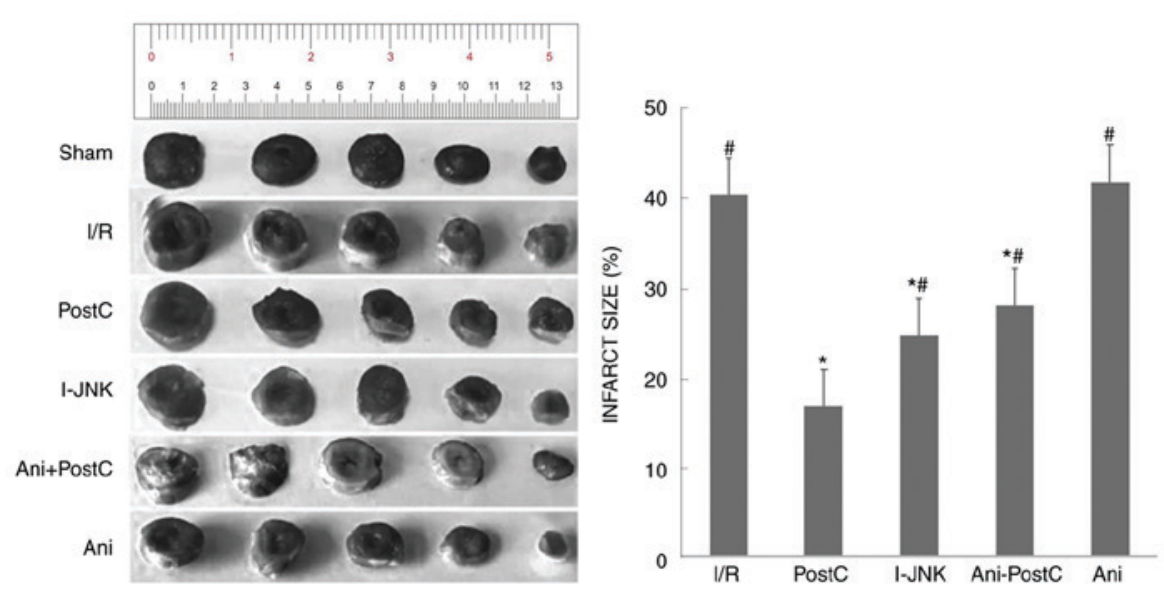

Figure 2. Infarct size in different groups. From left to right, cardiac slices are ordered from the base to the apex. Representative images of cross-sections of triphenyltetrazolium chloride stained ischemic hearts in the different groups. Relative infarct size expressed as the ratio of the infarct area to the total left ventricular volume. The results are presented as the mean \pm standard deviation. ${ }^{*} \mathrm{P}<0.05$ vs. the $\mathrm{I} / \mathrm{R}$ group; ${ }^{\mathrm{P}} \mathrm{P}<0.05$ vs. the post-conditioning group. I/R, ischemia reperfusion; Ani, anisomycin, postC, postconditioning; I-JNK, inhibitor of c-Jun N-terminal kinase. The infarct size in the Sham group was set as $0 \%$.

reperfusion, respectively (Table II). Ischemia-reperfusion injury led to a significant increase in PLA level 60 min and $3 \mathrm{~h}$ following reperfusion compared with the post-conditioning group $(\mathrm{P}<0.05)$. By contrast, ischemic postconditioning or application of the JNK inhibitor, SP600125, prevented the rise of PLA level at $60 \mathrm{~min}$ and $3 \mathrm{~h}$ post-reperfusion compared with the I/R injury group $(\mathrm{P}<0.05)$. Neither anisomycin treatment nor anisomycin with postconditioning significantly affected PLA level compared with ischemia-reperfusion conditioning (Table II and Fig. 3).

Finally, p-JNK expression was measured (Fig. 4). Compared with the I/R group, postconditioning or SP600125 significantly reduced $\mathrm{p}-\mathrm{JNK}$ expression $(\mathrm{P}<0.05)$, while treatment with anisomycin alone significantly increased p-JNK expression $(\mathrm{P}<0.05)$. The treatment with anisomysin with postconditioning partially also reduced the level of PLA.

\section{Discussion}

Using an ischemia-reperfusion model in rat hearts, ischemic postconditioning reduced infarct size and prevented the rise in TnI, CK-MB, p-JNK expression and the levels of PLA. These protective effects were similarly observed using the JNK inhibitor SP600125, but the effects were abolished with the JNK activator, anisomycin.

Ischemic postconditioning is an important cardiac protective mechanism, which reduces myocardial infarct size (12), the occurrence of arrhythmias (13), and improves vascular endothelial function (14) and myocardial systolic function. In ischemia-reperfusion injury, inflammation has a critical role in mediating myocardial damage. This involves the recruitment of leukocytes and the activation of platelets, leading to enhanced interactions between P-selectin glycoprotein ligand-1, which is expressed in the leukocytes, with P-selectin, which is expressed in the platelets to form PLAs. PLA is a sensitive index of thrombus load and inflammatory burden in vivo (1). Previous studies have demonstrated that high levels of PLA were not only positively and significantly associated with the risk of acute coronary syndrome (15) but were also closely associated with the no-reflow phenomenon (16).
Postconditioning is an important mechanism that protects against myocardial damage, reducing damage that is mediated by ischemia-reperfusion and improving cardiac contractile function. However, the molecular pathways responsible for protection against myocardial damage have not been entirely elucidated.

JNK is a member of the MAPK family that modulates multiple cellular functions, including proliferation, differentiation and apoptosis (17). JNK can be activated by ischemia and reperfusion insult. In the absence of JNK, mouse hearts subjected to ischemia-reperfusion have significantly less necrosis and apoptosis (18). On the contrary, the inhibition of JNK reduced the apoptosis of cardiomyocytes and infarct size following ischemia-reperfusion injury (19).

Nuclear factor (NF)- $\mathrm{\kappa B}$ is an important transcription factor that is involved in ischemia and reperfusion injury. A recent study demonstrated that intrinsic activation of AMP-activated protein kinase modulated JNK-NF- $\kappa B$ signaling cascade during hypoxia and reoxygenation stress conditions. It was critical to prevent excess mitochondrial reactive oxygen production and consequent JNK signaling during reperfusion, thereby protecting against mitochondrial permeability transition pore opening, irreversible mitochondrial damage and myocardial injury (20). In the present study, at different time points of MIRI, the level of PLA gradually increased. Compared with the injury-reperfusion group, the level of PLA in the PostC and I-JNK groups was significantly reduced at $60 \mathrm{~min}$ and $3 \mathrm{~h}$ following reperfusion, which suggested that PLA (a marker of inflammation and thrombosis) was involved in the pathogenesis of MIRI. Ischemic postconditioning or inhibition of JNK reduced the level of PLA. In addition, ischemia-reperfusion injury was able to increase p-JNK expression, which was prevented by ischemic postconditioning or JNK inhibition. This result suggested that myocardial ischemia-reperfusion was able to activate the JNK signaling pathway. Ischemic postconditioning or the inhibition of JNK served a critical role in inhibiting the inflammatory response during the ischemia/reperfusion process. Based on the above results, it was speculated that the cardioprotective effect of ischemic postconditioning may 
Table I. Serum markers and infarct area in all groups.

\begin{tabular}{|c|c|c|c|}
\hline Group & CK-MB (pg/ml) & $\operatorname{TnI}(\mathrm{pg} / \mathrm{ml})$ & Infarct area $(\%)$ \\
\hline Sham & $166.02 \pm 33.26^{\mathrm{a}, \mathrm{b}}$ & $7.79 \pm 0.54^{\mathrm{a}, \mathrm{b}}$ & $0.00 \pm 0.00^{\mathrm{a}, \mathrm{b}}$ \\
\hline Ischemia-reperfusion & $637.31 \pm 43.99^{\mathrm{b}}$ & $19.67 \pm 0.66^{\mathrm{b}}$ & $40.17 \pm 1.77^{\mathrm{b}}$ \\
\hline Postconditioning & $257.38 \pm 53.12^{\mathrm{a}}$ & $10.05 \pm 0.81^{\mathrm{a}}$ & $16.68 \pm 2.06^{\mathrm{a}}$ \\
\hline JNK inhibitor SP600125 & $402.10 \pm 47.94^{\mathrm{a}, \mathrm{b}}$ & $14.26 \pm 1.31^{\mathrm{a}, \mathrm{b}}$ & $24.62 \pm 1.58^{\mathrm{a}, \mathrm{b}}$ \\
\hline JNK activator anisomycin and postconditioning & $500.84 \pm 52.93^{\mathrm{a}, \mathrm{b}}$ & $17.32 \pm 0.77^{\mathrm{a}, \mathrm{b}}$ & $27.91 \pm 1.79^{\mathrm{a}, \mathrm{b}}$ \\
\hline JNK activator anisomycin & $638.34 \pm 25.87^{\mathrm{b}}$ & $19.96 \pm 0.72^{b}$ & $40.62 \pm 1.59^{\mathrm{b}}$ \\
\hline
\end{tabular}

${ }^{\mathrm{a}} \mathrm{P}<0.05$ vs. the $\mathrm{I} / \mathrm{R}$ group; ${ }^{\mathrm{b}} \mathrm{P}<0.05$ vs. the post-conditioning group. CK-MB, creatine kinase muscle/bone; TnI, troponin I; JNK, c-Jun $\mathrm{N}$-terminal kinase.

Table II. Expression of platelet-lymphocyte aggregates at different time points.

\begin{tabular}{|c|c|c|c|c|c|}
\hline Group & Baseline & $\begin{array}{l}30 \text { min following } \\
\text { ischemia }\end{array}$ & $\begin{array}{l}30 \text { min following } \\
\text { reperfusion }\end{array}$ & $\begin{array}{l}60 \text { min following } \\
\text { reperfusion }\end{array}$ & $\begin{array}{l}3 \mathrm{~h} \text { following } \\
\text { reperfusion }\end{array}$ \\
\hline Sham & $7.96 \pm 0.30$ & $8.70 \pm 0.56^{\mathrm{a}, \mathrm{b}}$ & $8.99 \pm 0.56^{\mathrm{a}, \mathrm{b}}$ & $8.80 \pm 0.36^{\mathrm{a}, \mathrm{b}}$ & $10.11 \pm 0.87^{\mathrm{a}, \mathrm{b}}$ \\
\hline Ischemia-reperfusion & $8.06 \pm 0.23$ & $23.70 \pm 2.79$ & $34.78 \pm 2.79$ & $42.00 \pm 2.02^{\mathrm{b}}$ & $51.03 \pm 2.02^{\mathrm{b}}$ \\
\hline Postconditioning & $9.01 \pm 0.26$ & $22.76 \pm 1.93$ & $32.50 \pm 1.93$ & $23.90 \pm 0.89^{\mathrm{a}}$ & $18.40 \pm 1.27^{\mathrm{a}}$ \\
\hline JNK inhibitor SP600125 & $8.43 \pm 0.22$ & $23.90 \pm 2.06$ & $32.90 \pm 2.06$ & $29.57 \pm 1.46^{\mathrm{a}, \mathrm{b}}$ & $26.64 \pm 1.18^{\mathrm{a}, \mathrm{b}}$ \\
\hline $\begin{array}{l}\text { JNK activator anisomycin } \\
\text { and postconditioning }\end{array}$ & $9.30 \pm 0.29$ & $21.81 \pm 2.14$ & $32.69 \pm 2.14$ & $45.66 \pm 0.94^{\mathrm{b}}$ & $54.35 \pm 1.45^{\mathrm{b}}$ \\
\hline JNK activator anisomycin & $9.23 \pm 0.40$ & $22.20 \pm 2.82$ & $32.7 \pm 2.82$ & $45.70 \pm 1.69^{b}$ & $54.80 \pm 1.40^{\mathrm{b}}$ \\
\hline
\end{tabular}

${ }^{a} \mathrm{P}<0.05$ vs. the $\mathrm{I} / \mathrm{R}$ group; ${ }^{\mathrm{b}} \mathrm{P}<0.05$ vs. the post-conditioning group. JNK, c-Jun $\mathrm{N}$-terminal kinase.

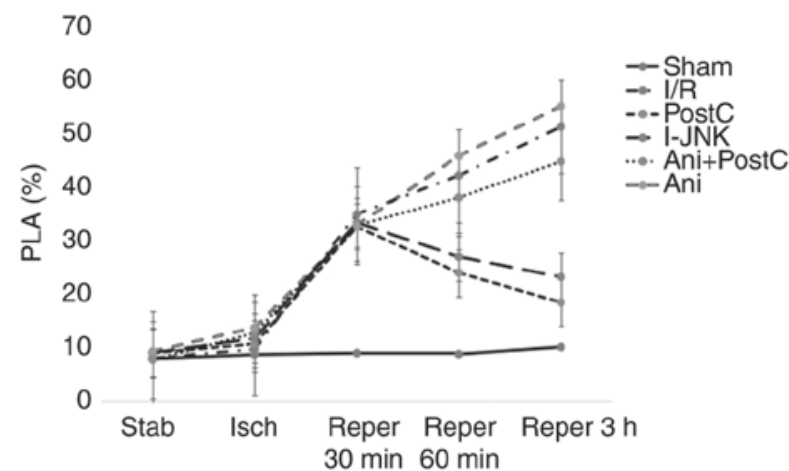

Figure 3. The level of PLAs at different time points following ischemia or reper in different groups. CD45 and CD41-Phycoerythrin were used to detect PLAs levels in each group. At the time point of baseline, 30 min following ischemia, 30, $60 \mathrm{~min}$ and $3 \mathrm{~h}$ following reper, the level of PLAs was detected by flow cytometry. Reper, reperfusion; CD, cluster of differentiation; I/R, ischemia reperfusion; Ani, anisomycin, postC, postconditioning; I-JNK, inhibitor of c-Jun N-terminal kinase; PLA, platelet-lymphocyte aggregates.

be associated with the attenuation of inflammation, which occurred during ischemia/reperfusion via the modulation of the JNK-mediated NF- $\mathrm{B}$ signaling pathway. Therefore, regulating $\mathrm{JNK}$ activation may represent an important novel strategy in the prevention and treatment of MIRI.

Although the current study provided interesting results, there remain certain weaknesses. Firstly, MIRI is a complicated pathological process, however, only JNK MAPK

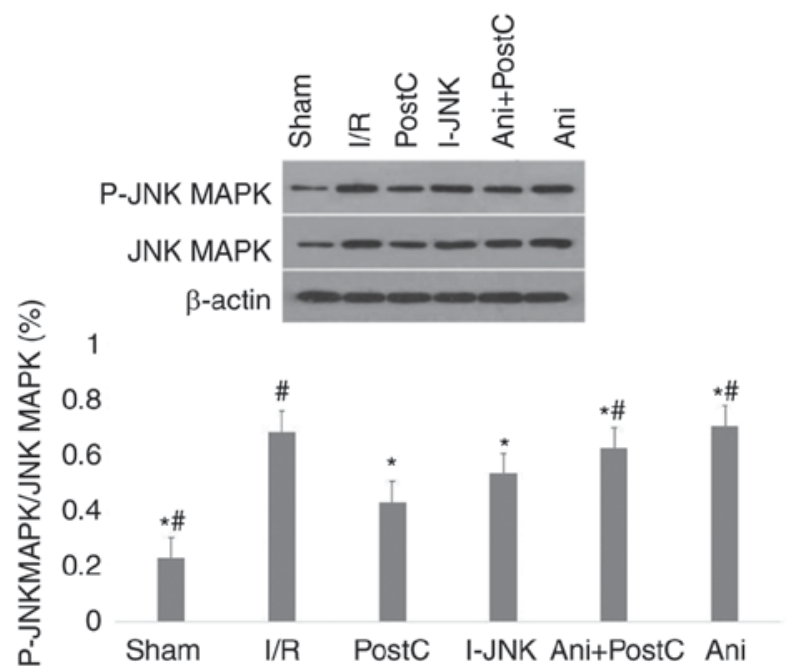

Figure 4. Expression of P-JNK MAPK in different groups. Western blot analysis of the expression of P-JNK MAPK in the sham, ischemia-reperfusion $(\mathrm{I} / \mathrm{R})$, ischemic PostC, I-JNK, Ani and ischemic postconditioning with anisomycin (Ani+PostC) groups. Statistical analysis of levels of protein expression. ${ }^{*} \mathrm{P}<0.05$ vs. the $\mathrm{I} / \mathrm{R}$ group; ${ }^{~} \mathrm{P}<0.05$ vs. the post-conditioning group. I/R, ischemia reperfusion; Ani, anisomycin, postC, postconditioning; I-JNK, inhibitor of c-Jun N-terminal kinase; $\mathrm{p}$, phosphorylated.

signaling pathway was included in the present study, the whole signaling network was not investigated. Secondly, the infarct size in I-JNK group was significantly reduced, but 
the expression level of apoptosis associated proteins was not detected. Finally, although pharmacological inhibition of JNK has cardioprotective effects, it is not clear whether this JNK inhibitory effect will produce adverse effects in other tissues.

JNK is a critical mediator of MIRI. Ischemic postconditioning can reduce the level of PLA during reperfusion by inhibiting the phosphorylation of JNK MAPK, thereby reducing MIRI. Pharmacological inhibition and activation of JNK can improve and reduce the cardioprotective effects, respectively. The results of the present study help to explain the mechanism of the cardioprotection against MIRI of postconditioning in rats. In addition, it provided novel insight and targets for the therapeutic strategy of MIRI. JNK inhibition may represent a novel therapeutic strategy for the alleviation of MIRI.

\section{Acknowledgements}

Not applicable.

\section{Funding}

The present study was supported by grants from the National Natural Science Foundation of China (grant no. 81500271).

\section{Availability of data and materials}

All data generated or analyzed during this study are included in this published article.

\section{Authors' contributions}

FR, NM and MG performed experiments and drafted the manuscript. $\mathrm{CZ}$ and $\mathrm{XS}$ performed the experiments. LL and TL analyzed the data. JL and JS performed the animal experiments. MD and GT made substantial contributions to the design of the present study. All authors approved the final version of manuscript.

\section{Ethics approval and consent to participate}

The present study was approved by the Research Commission on Ethics of the Affiliated Yantai Yuhuangding Hospital of Qingdao University.

\section{Patient consent for publication}

Not applicable.

\section{Competing interests}

The authors declare they have no competing interests.

\section{References}

1. Bonvini RF, Hendiri T and Camenzind E: Inflammatory response post-myocardial infarction and reperfusion: A new therapeutic target? European Heart J Suppl 7 (suppl_I): I27-I36, 2005.

2. Schächinger V, Britten MB and Zeiher AM: Prognostic impact of coronary vasodilator dysfunction on adverse long-term outcome of coronary heart disease. Circulation 101: 1899-1906, 2000.
3. Furman MI, Barnard MR, Krueger LA, Fox ML, Shilale EA, Lessard DM, Marchese P, Frelinger AL III, Goldberg RJ and Michelson AD: Circulating monocyte-platelet aggregates are an early marker of acute myocardial infarction. J Am Coll Cardiol 38: 1002-1006, 2001.

4. Faraday N, Braunstein JB, Heldman AW, Bolton ED, Chiles KA, Gerstenblith G and Schulman SP: Prospective evaluation of the relationship between platelet-leukocyte conjugate formation and recurrent myocardial ischemia in patients with acute coronary syndromes. Platelets 15: 9-14, 2004.

5. Thuny F, Lairez O, Roubille F, Mewton N, Rioufol G, Sportouch C, Sanchez I, Bergerot C, Thibault H, Cung TT, et al: Post-conditioning reduces infarct size and edema in patients with ST-segment elevation myocardial infarction. J Am Coll Cardiol 59: 2175-2181, 2012.

6. Xue F, Yang X, Zhang B, Zhao C, Song J, Jiang T and Jiang W: Postconditioning the human heart in percutaneous coronary intervention. Clin Cardiol 33: 439-444, 2010.

7. Huang C, Jacobson K and Schaller MD: A role for JNK-paxillin signaling in cell migration. Cell Cycle 3: 4-6, 2004.

8. Uehara T, Bennett B, Sakata ST, Satoh Y, Bilter GK, Westwick JK and Brenner DA: JNK mediates hepatic ischemia reperfusion injury. J Hepatol 42: 850-859, 2005.

9. Wang Z, Tang B, Tang F, Li Y, Zhang G, Zhong L, Dong C and He S: Protection of rat intestinal epithelial cells from ischemia/reperfusion injury by (D-Ala2, D-Leu5)-enkephalin through inhibition of the MKK 7-JNK signaling pathway. Mol Med Rep 12: 4079-4088, 2015.

10. Yang DH, Tan N, He PC, Liu Y, Wen JY, Chen JY, Zhou YL and Huang WH: Increased platelet-leukocyte aggregates in patients with acute coronary syndrome. Zhonghua Xin Xue Guan Bing Za Zhi 40: 482-486, 2012 (In Chinese).

11. Li Y, Ge X and Liu X: The cardioprotective effect of postconditioning is mediated by ARC through inhibiting mitochondrial apoptotic pathway. Apoptosis 14: 164-172, 2009.

12. Staat P, Rioufo TG, Piot C, Cottin Y, Cung TT, L'Huillier I, Aupetit JF, Bonnefoy E, Finet G, André-Fouët X and Ovize M: Postconditioning the human heart. Circulation 112: 2143-2148, 2005.

13. Kloner RA, Dow J and Bhandari A: Postconditioning markedly attenuates ventricular arrhythmias after ischemia-reperfusion. J Cardiovasc Pharmacol Ther 11: 55-63, 2006.

14. Yang DH, Tan N, He PC, Liu Y, Wen JY, Chen JY, Zhou YL and Huang WH: Increased platelet-leukocyte aggregates in patients with acute coronary syndrome. Zhonghua Xin Xue Guan Bing Za Zhi 40: 482-486, 2012 (In Chinese).

15. Sivaraman V, Mudalagiri NR, Di Salvo C, Kolvekar S, Hayward M, Yap J, Keogh B, Hausenloy DJ and Yellon DM: Postconditioning protects human atrial muscle through the activation of the RISK pathway. Basic Res Cardiol 102: 453-459, 2007.

16. Ren F, Mu N, Zhang X, Tan J, Li L, Zhang C and Dong M: Increased platelet-leukocyte aggregates are associated with myocardial no-reflow in patients with ST elevation myocardial infarction. Am J Med Sci 352: 261-266, 2016.

17. Weston CR and Davis RJ: The JNK signal transduction pathway. Curr Opin Cell Biol 19: 142-149, 2007.

18. Kaiser RA, Liang Q, Bueno O, Huang Y, Lackey T, Klevitsky R, Hewett TE and Molkentin JD: Genetic inhibition or activation of JNK $1 / 2$ protects the myocardium from ischemia-reperfusion-induced cell death in vivo. J Biol Chem 280: 32602-32608, 2005.

19. Ferrandi C, Ballerio R, Gaillard P, Giachetti C, Carboni S, Vitte PA, Gotteland JP and Cirillo R: Inhibition of c-Jun $\mathrm{N}$-terminal kinase decreases cardimoyocyte apoptosis and infarct size after myocardial ischemia and reperfusion in anaesthetized rats. Br J Pharmacol 142: 953-960, 2004.

20. Zaha VG, Qi D, Su KN, Palmeri M, Lee HY, Hu X, Wu X, Shulman GI, Rabinovitch PS, Russell RR III and Young LH: AMPK is critical for mitochondrial function during reperfusion after myocardial ischemia. J Mol Cell Cardiol 91: 104-113, 2016. 